\title{
Assumption and Criticism on RNA World Hypothesis from Ribozymes to Functional Cells
}

\author{
Sami EL Khatib*, Ahmad Raslan \\ Department of Biological Sciences, School of Arts \& Sciences, Lebanese International University, Lebanon
}

Received December 27, 2020; Revised March 2, 2021; Accepted March 12, 2021

\section{Cite This Paper in the following Citation Styles}

(a): [1] Sami EL Khatib, Ahmad Raslan, "Assumption and Criticism on RNA World Hypothesis from Ribozymes to Functional Cells," Bioengineering and Bioscience, Vol. 8, No. 1, pp. 1 - 12, 2021. DOI: 10.13189/bb.2021.080101.

(b): Sami EL Khatib, Ahmad Raslan (2021). Assumption and Criticism on RNA World Hypothesis from Ribozymes to Functional Cells. Bioengineering and Bioscience, 8(1), 1 - 12. DOI: 10.13189/bb.2021.080101.

Copyright $\bigcirc 2021$ by authors, all rights reserved. Authors agree that this article remains permanently open access under the terms of the Creative Commons Attribution License 4.0 International License

\begin{abstract}
The origin of life has long baffled scientists, trying to shed some light on the universe of the unknown we live in. The RNA world hypothesis is merely an idea that tries to grasp the root of life by going beyond phylogenetic trees, and the 'Drawinian threshold'. The birth of this hypothesis came with the discovery of ribozymes and certain relic molecules left behind by an RNA ruled world about -3.8 billion years ago. These primitive cells were the most minimal forms of life and experiments are trying to show this by mimicking primitive earth environments. Nucleotides have been shown to polymerize into random chains easily on clay or in ice and the chemical synthesis of RNA is possible although extremely challenging without the modern cellular machinery. The RNA world is untraceable because of horizontal gene transfer, which is highly criticized by researches, and still remains a widely researched and studied topic as it could be the heart of life on other earth like planets in the universe. The state of the model needs improvements in analytical techniques and experiments for the rate of Horizontal Gene Transfer on loosely bound components, and continued comparison of the different types of phylogenetic trees to widen the evolution of the genetic core. Furthermore the development of analytical techniques used for the monitoring of the Donan effect may show more insight into the division and growth of primitive cellular membranes and the continued research and exploration in the fields of Ribozymes, with a goal to reach a higher catalytic range with high fidelity and rate.
\end{abstract}

Keywords The RNA World, Darwinian Threshold, Horizontal Gene Transfer, Relics of the RNA World, Ribozymes, Group I Introns, Primitive Cell Membranes,
Phosphoramidites, the Donan Effect

\section{The Birth of the RNA World Hypothesis}

The elementary hypothesis was first described by Leslie Orgel, Carl Woese, and Francis Crick in the 1960's, articles written about the RNA world hypothesis are highly unusual in format and style, and are difficult to be classified under any scientific publication, Woese himself refers to his work as a 'genetic annealing model for the universal ancestor of all extant life' [1]. Woese's analysis is qualitative rather than quantitative, general rather than specific, and does not strain to make concrete predictions (hence unfalsifiable). Despite the lack of formulas, plots and schematics the hypothesis more or less fits the standard perception of a theory and model, because of the same generality that allows for a diverse family of models to fall under this framework [1], [2].

In order to fully understand the vital mechanisms occurring in current living organisms, there is a necessity to shed the light on the way they developed during the process of evolution. The central concern of these issues could be coined to the mechanism throughout of which the expression of genetic information takes place, which necessitates extremely complex machinery that proceeds from DNA as a carrier of hereditary information to the functional protein passing through an RNA intermediate molecule. Many questions, on perspective, could be focused on how did this complex machinery arise? Under 
which conditions could the RNA molecule be synthesized and maintain its stability? What is(are) the reasons that the RNA has been designated as a functional compound serving the main genetic and associated catalytic processes, rather than other alternative polymers? How does the origin of replicating systems relate to the origin of protocells and metabolism? How complex did the RNA world become before the advent of the genetic code and translation?

\section{Phylogenetics and the Darwnian Threshold}

The evolution of modern cells is arguably the most challenging and important problem faced in the field of biology. A universal phylogenetic tree (Figure 1) depicts the modern types of cells with a common ancestral root which is determined based on molecular (rRNA) sequences in a canonical pattern [3], meaning they are so different from one another but still share a common intersection of origin. This led to the Darwinian thinking of endosymbiosis, or cell fusion, to produce a higher functioning organism, explaining the origin of eukaryotic nucleuses and the origin of mitochondria and chloroplasts, but to address the actual cellular root of the universal phylogenetic tree (depicted by the horizontal line in Figure 1), one must assume that its transcriptional state would resemble that of a modern cell, which is clearly not the case [3], [4].

The RNA world hypothesis must be viewed out of Darwinian/Endosymbiosis concepts, thus individual lineages, species as we know them today, emerged from this common ancestral disorder, only when cellular organization reached a certain degree of complexity and connectedness, this transition is marked by key functions evolving (such as translation), and not being lost (by Horizontal Gene Transfer) in the primitive or proto cell. The stage at which this new more complex, integrated organization occurs has been termed the 'Darwinian threshold' [1] and the RNA world lie behind it.

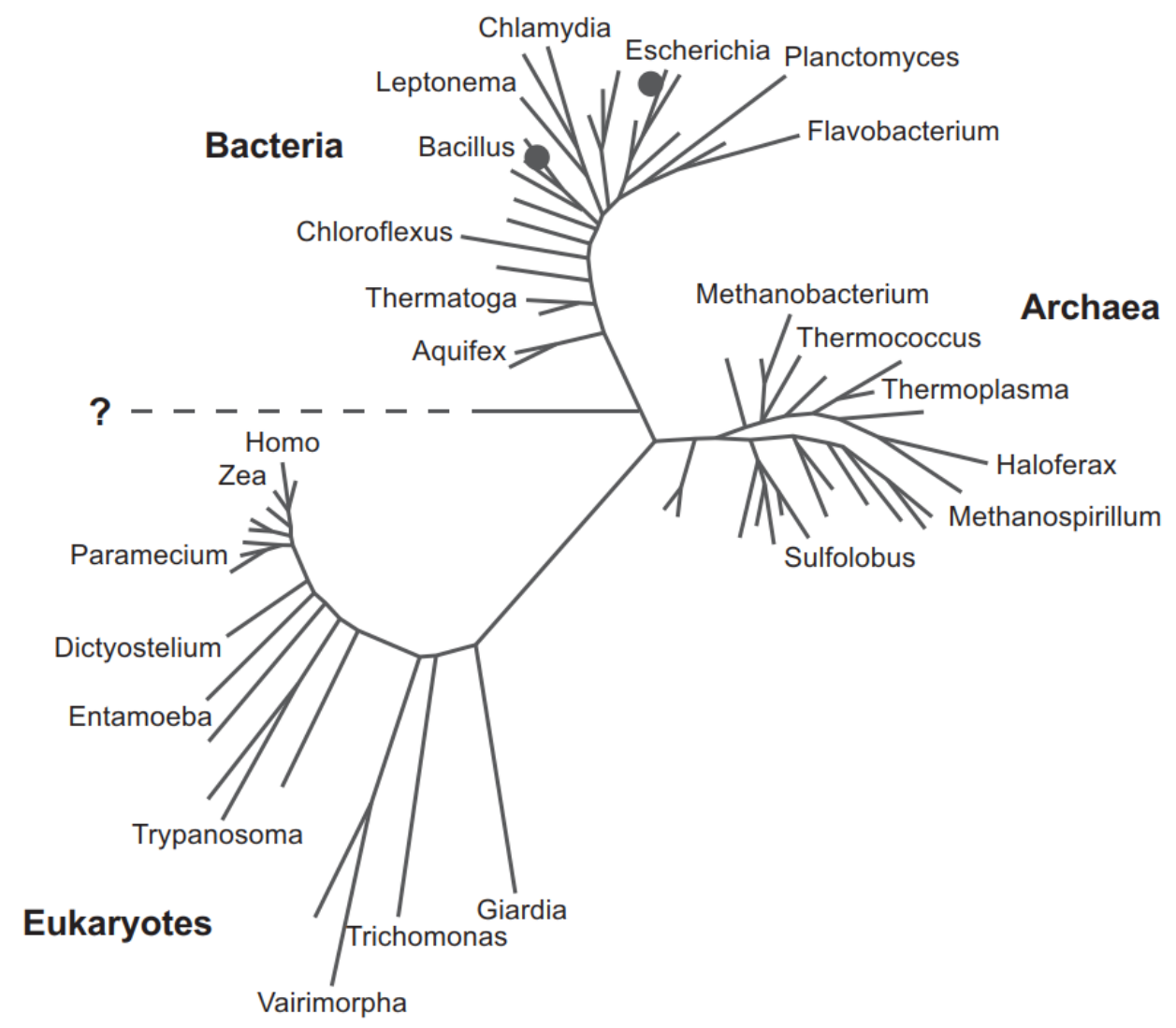

Figure 1. The universal phylogenetic tree as determined by ribosomal RNA sequences analyses; Root depicted by horizontal line [4] 

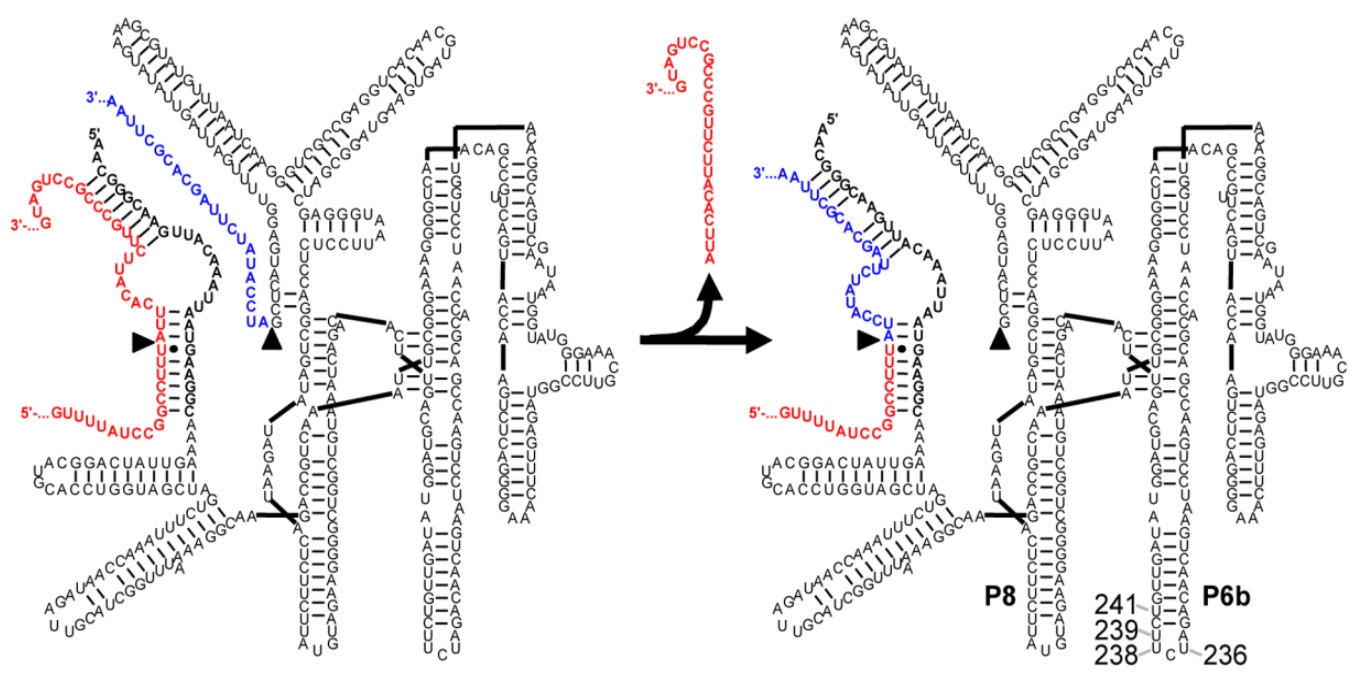

Figure 2. Structures and functions of a Ribozyme; Secondary structure of a trans-splicing group I intron ribozyme; the ribozyme (black) is base-paired to the target site of the mutated CAT mRNA (red). The 5'-splice site and the 3'-splice site are indicated with arrowheads. During the trans-splicing reaction (arrows from left to right) the 3'-exon of the ribozyme (blue) replaces the 3'-portion of the mutated CAT mRNA leading to the repaired CAT mRNA (red/blue). The positions of the P8 and the P6b stem-loops in the ribozyme are indicated, together with the four positions 236, 238, 239, and $241[7]$<smiles>CC(=O)SCCNC(=O)CCNC(=O)[C@@H](O)C(C)(C)CO[P@](=O)(O)O[P@](=O)(O)OC[C@H]1O[C@@H](n2cnc3c(N)ncnc32)[C@H](O)[C@@H]1OP(=O)(O)O</smiles>

Figure 3. Structure of Acetyl-CoA; depicting a functional thioester bond and a nonfunctional phosphorylated ADP (Created by RCSB Protein Data Bank ID: ACO) [8]

\section{Ribozymes and Relic Molecules}

The rudimentary idea wasn't taken into consideration until the discovery of Ribozymes; group I introns (Figure 2) and RNase P in the 1980's, by Tom Cech and Sid Altman respectively. These discoveries led to the foundation and research of the RNA world hypothesis [2], [3].

Examples of small self-cleaving RNAs embedded in noncoding regions already have been found to be involved in the control of gene expression, although their origin remains uncertain. The wide spread of the Hammer Head Ribozyme (HHR) motifs studied by De La Pena and Garcia-Robles in 2010, among genomes from Bacteria, Chromalveolata, Plantae, and Metazoa kingdoms, showed intergenic HHRs in three different bacterial genomes, whereas metagenomic data from the Galapagos islands showed the occurrence of similar ribozymes that could be regarded as direct relics of the RNA world. Among Eukaryotes, HHRs were detected in the genomes of three water molds as well as 20 plant species, ranging from unicellular algae to vascular plants. These HHRs were very similar to those found in small RNA plant pathogens, and in some cases appeared as close tandem repetitions. In metazoans a parallel situation of tandemly repeated HHR motifs was also detected from cnidarians to invertebrates, with special emphasis among hematophagous and parasitic organisms [5], [6]. These results conclude the wide spread of the HHR in all life forms.

Relic molecules, such as Acetyl-CoA (Figure 3) have a thioester bond as the working part of the molecule, and at the end of the molecule for no apparent reason is a nucleotide, thought to have assisted in the holding of the molecule by primitive ribozymes [8].

Filippova in 2014 showed the crystal structure of Acetyl-CoA bound to N-Acyl Transferase and Sulfate 
ions, and found that the nucleotide has no affinity to the complex at all (Figure 4) [7], [8].

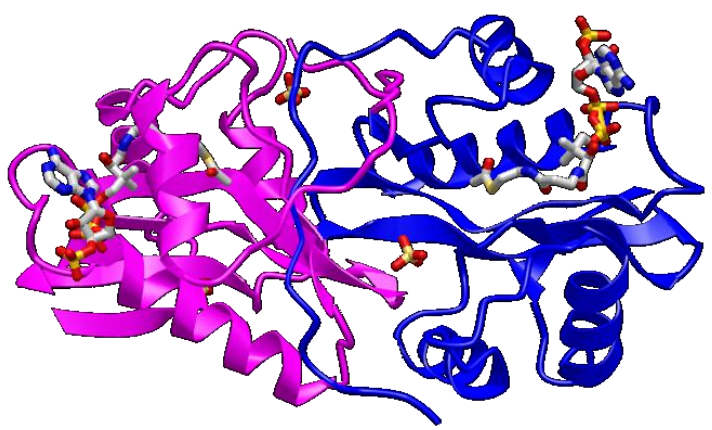

Figure 4. Crystal structure of N-Acyltransferase in complex with Acetyl-CoA and Sulphate ions from Escherichia coli; protein chains are colored from $\mathrm{N}$ terminal to the $\mathrm{C}$ terminal using a rainbow (spectral) gradient, the binding of the sulphate ions shows that it is the thioester which complexes. The nucleotide is shown to have no affiliation in the complex [8]

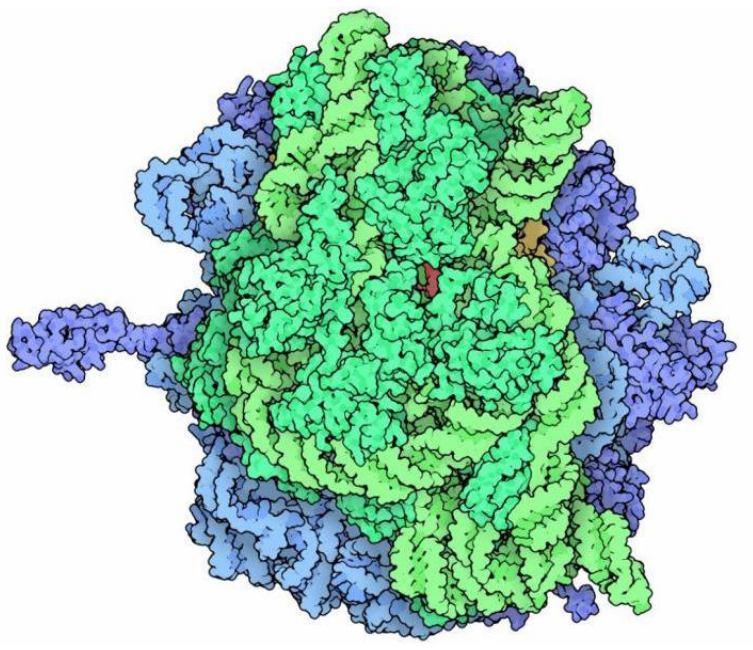

Figure 5. Structure of the 70s Ribosome; Frontal view: Protein compositions are indicated in blue, RNA compositions are indicated in green, and core activation site formed from a ribozyme indicated in yellow and red [10]
Further evidences that led to the RNA world hypothesis also include the synthesis of DNA; the path way taken to produce dNTPs to be incorporated to new strands is not De Novo as you would think, but rather that dNTPs are made from already existing RNA molecules, and then deoxygenated, indicating that DNA evolved to be the sole information holder from RNA, and RNA while still having information capacity evolved into more functional roles (mRNA, tRNA, rRNA, siRNA) [9]. Perhaps the most compelling evidence is the structure of the ribosome (Figure 5), which is an essential component in the synthesis of proteins, which underline most of the modern biochemical metabolisms in the modern cell, and is the sole basis of the known phylogenetic tree branches, it has been shown that at the heart of the ribosome (active site) is a ribozyme [10].

\section{Proteins and Membranes in the Time Line of the RNA World}

\section{Time Line}

When the RNA world would have come to life, what is the RNA world's time frame? The first evidence for modern microbial life is roughly 3.5 billion years ago and geological formation of earth is estimated to be at 4.5 billion years ago, so there is an approximate billion year interval between the cooling of the planet, creation of the hydrosphere, and the first concrete evidence of microbial life, during this billion year interval it is thought that prebiotic chemistry would undergo complex transitions in harsh and different environments that would give rise to the pre-RNA world that could start to carry out biochemical functions in primitive cells and then would eventually lead to the emergence of much more complicated cells that are more biochemically similar to modern life today [11] [12] (Figure 6).
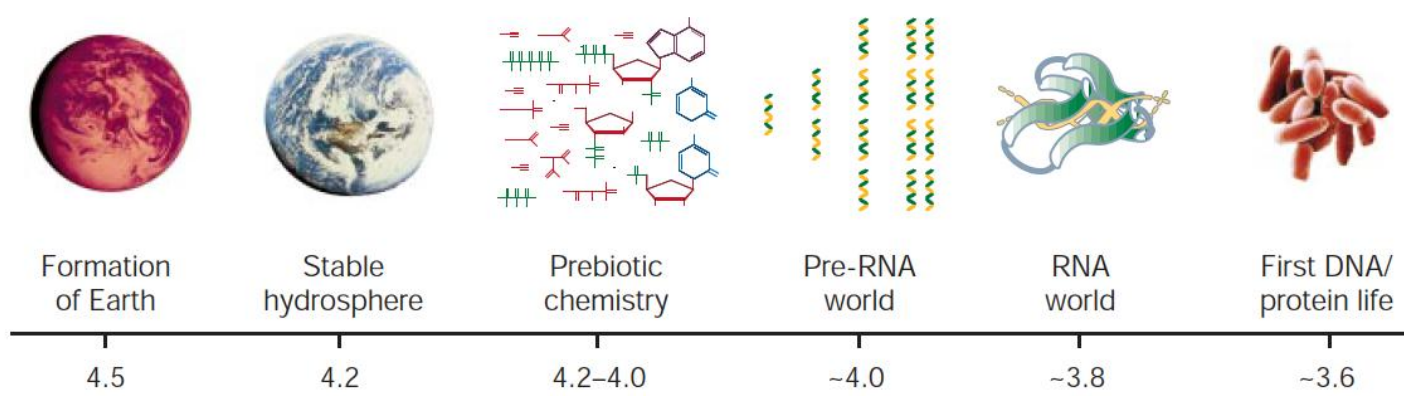

Figure 6. Time Frame; Time Line of events pertaining to the early history of life on Earth, with approximate dates in billions of years before the present [11] 


\section{Protein Life}

Between 3.6 and 3.8 billion years, lies the era of the first DNA/protein life, but how did the 'invention' of DNA and proteins arise from the RNA world? DNA from the RNA world can be understood as RNA, which can be reversely transcribed into DNA under the mechanisms of template-directed synthesis using base pairing. This is very convenient to address DNA coming from the RNA world, but not so in the case of proteins [13].

In in vitro selection experiments, searching for ribozymes that have affinity to amino acids (aminoacids-aptamers), it was revealed that RNA could bind amino acids with significant specificity [14] (Geiger, 1996). So with RNA containing multiple amino acid binding sites, peptide bonds could form when the RNA structurally folds and brings the amino acids spatially adjacent [13] (Figure 7).

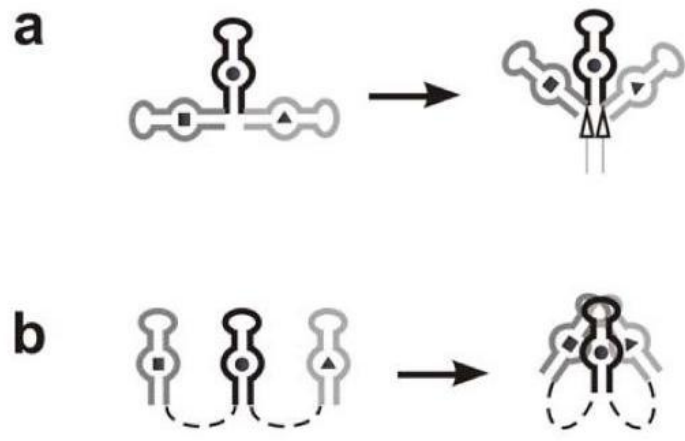

Figure 7. The stereo-chemical consideration of the structure of aa-aptamers for peptide synthesis; the dark gray, black and light gray portions of the RNAs correspond to the amino acid-binding sites for three different amino acids, the square, circle and triangle, respectively. (a) Depicts stereo-chemical limitation because of the organization of the aptamer one after the other. (b) The dashed line depicts spacer sequences which over-come the stereo-chemical limitations and allow the three amino acids to form peptide binds [13]

\section{Basic Assumptions of Molecular Composition}

Every researcher tends to think of the RNA world in a different way, so it would be ineffective to attempt a constrained definition, but all RNA world models include three basic assumptions: (i) At some time in the evolution of life, genetic continuity was assured by the replication of RNA; (ii) Watson-Crick base-pairing was the key to replication; (iii) genetically encoded proteins were not involved as catalysts [15].

Investigators differ in what they assume about life that may have preceded the RNA world; the pre-RNA world (Figure 6). They also debate about the metabolic complexity of the RNA world and about the role of small-molecule cofactors, possibly including peptides in the chemistry of the RNA world [15].

Assumptions from Joyce and Orgel in 1993 include the 'The Molecular Biologist's Dream', where one can imagine that all of the components of RNA were freely available in a prebiotic pool, and these components just assembled into polymers prior to the existence of any macromolecules [15]. However as Robertson and Joyce in 2012 explain this triggers 'The Prebiotic Chemist's Nightmare', so named because of the untraceable mixtures that are obtained in experiments designed to simulate the chemistry of primitive earth. The assumptions on the origin of the RNA world are far from being solved, and it is highly likely that RNA was also preceded by some other replicating, evolving molecule [15], [16], [17].

\section{Primitive-Cell Membranes}

What would the components of a primitive cell be living in the RNA world? When thinking of an RNA world, researchers minimalize components as to try to reach as far back in evolutionary time as possible, thus the components would be of only two (i) Nucleic Acid; RNA or precursor, that was completely dissolved in the aqueous compartment of a (ii) primitive-cell membrane which essentially creates the closed environment for the nucleic acid, excluding it from the entire universe outside [18] (Figure 8).

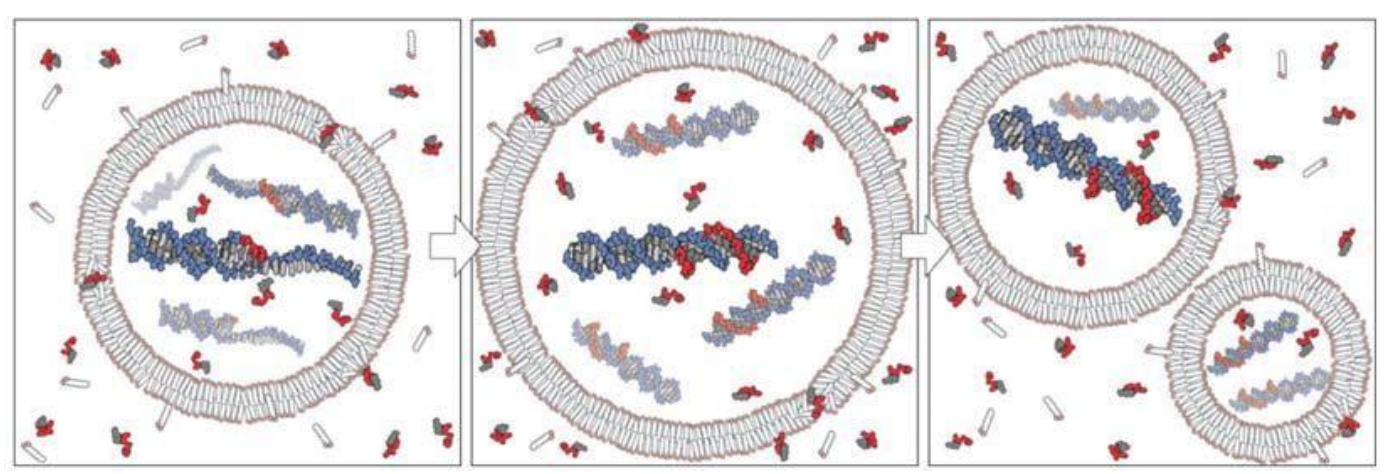

Figure 8. Schematic of a model primitive cell; Fatty acid bilayer and RNA (or precursors) are depicted (left). Growth of the protocell (middle) happens when equilibrating free nucleotides and lipids are incorporated into the cell. Division (right) is caused either physically or chemically [18] 

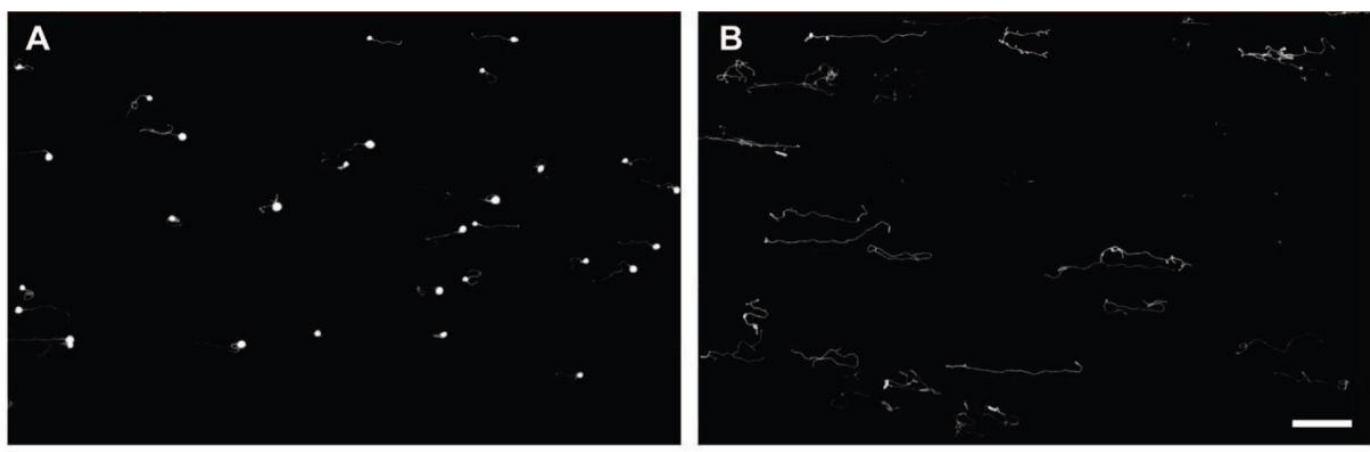

C

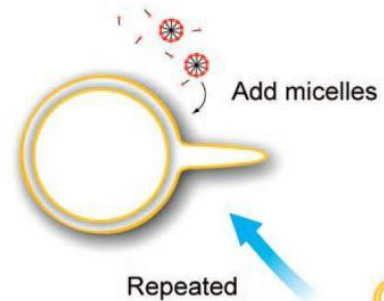

cycles
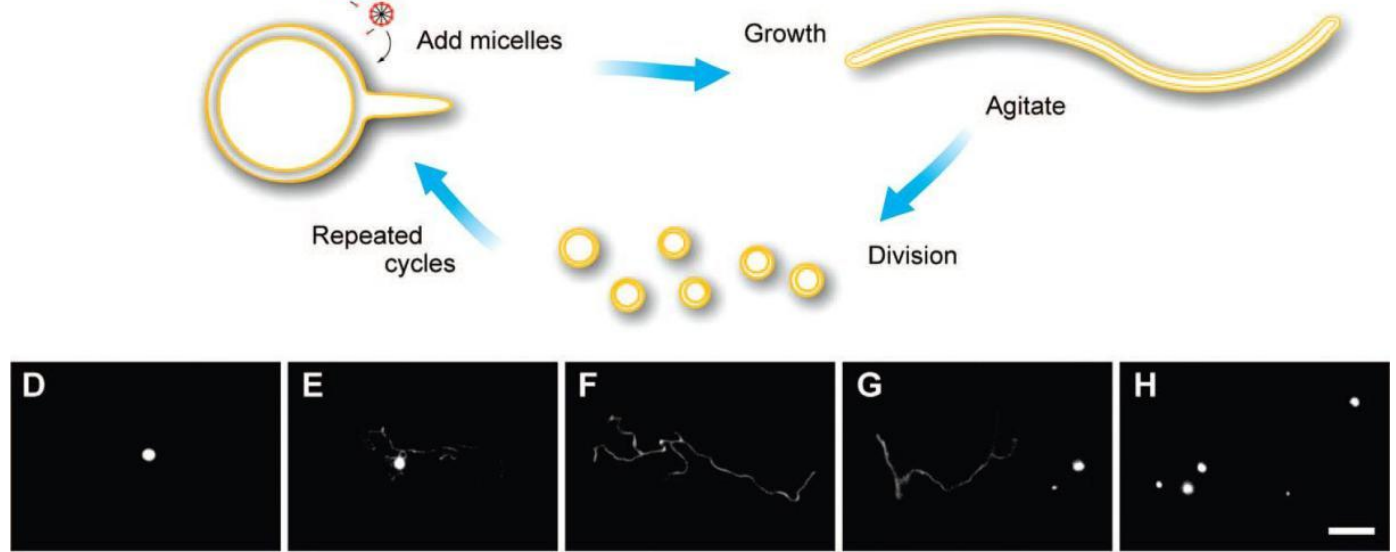

Figure 9. Vesicle growth and division; (A\&B) Epifluorescene micrographs of vesicle shape transformations during growth, 10 and 30 minutes after the addition of 5 equiv of oleate micelles to multilamellar oleate vesicles, at $\mathrm{pH}$ 8.5. (C) Schematic diagram of cyclic multilamellar vesicle growth and division. (D-F) Growth of a single multlamellar oleate vesicle at, 3 minutes, 10 minutes, and 25 minutes after the addition of 5 equiv of oleate micelles respectively. (G-H) shows the reformation of vesicles from the long growth tube after slight agitation [20]

Laboratory investigations show that fatty acids (like Oleic acid, Myristoteic acid or Capric acid, all abundantly naturally occurring with capric acid being the most prebiotically compatible) can form vesicles and micelles at alkaline $\mathrm{pH}$ [18], simply when shaken with some salt, these vesicles grow and divide as shown in Figure 9, they are highly dynamic and not stable sufficient, but when glycerol and alcohols are incorporated into the vesicle their stability and ability to not leak their contents drastically improves, so much that they could be boiled and not leak a single oligonucleotide [19] [6].

This incorporation of alcohols and/or glycerol also increases the polarity of the membrane allowing small polar items to pass in and not out, essentially trapping them, for incorporation into the growing RNA strand and membrane [19], [20].

Cell division could be driven by mechanical or chemical forces, such as osmotic pressure build up inside the cell caused by the stabilizing factors for RNA (and not RNA its self), which causes a strain on the growing membrane to incorporate more fatty acids from the environment essentially eating neighbor membranes, known as the Donan effect [19], and mechanical filters or forces that physically rip a membrane apart, but causes leakage of the contents, eventually leading to the reassembly of smaller vesicles [21], [22].

To address the hypothesis that phospholipids could drive competition between primitive cells (Donan effect), Budin and Szostak in 2011 tested whether mixed fatty acid- phospholipid vesicles grow when mixed with pure fatty acid vesicles. They monitored surface area change by measuring the Forster Resonance Energy Transfer (FRET) between donor and acceptor fluorophores included in a fixed initial concentration in the bilayer and found the results depicted in Figure 10 [19]. 

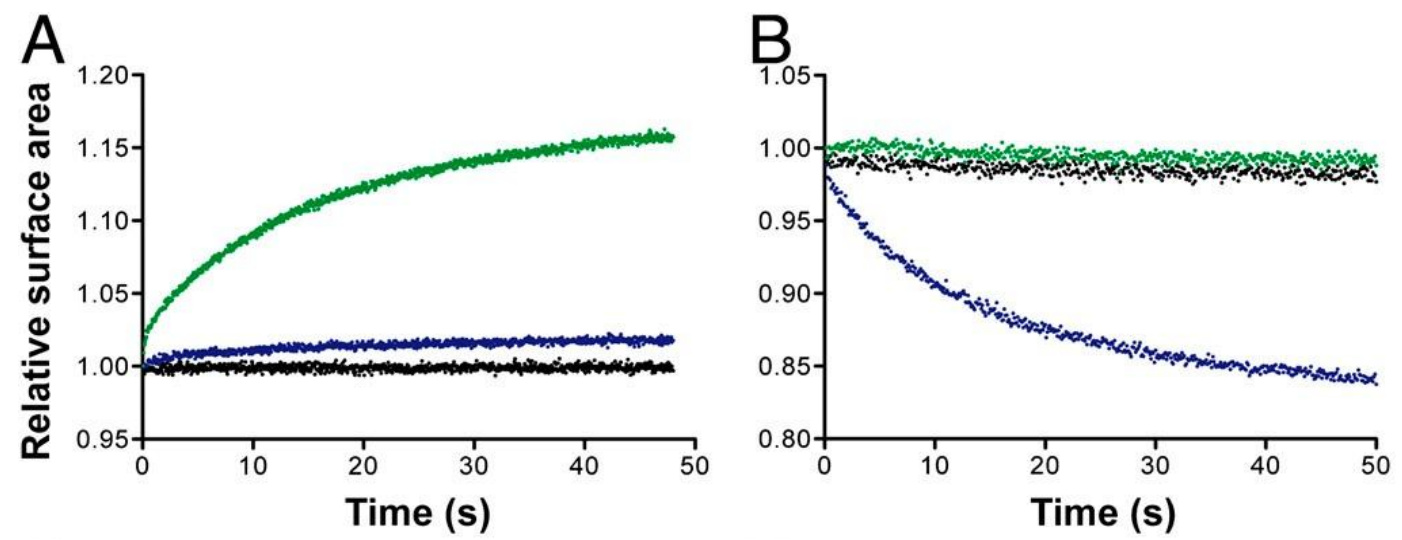

Figure 10. Phosopholipids drive competition between fatty acid vesicles; (A and B). Growth of FRET-labeled 90:10 oleate : DOPA* vesicles (A) and shrinkage of FRET-dye labeled oleate vesicles (B) when mixed 1:1 with buffer (black), unlabeled oleate vesicles (green), or unlabeled 90: 10 oleate : DOPA vesicles (blue) ('di-oleoyl-phosphatidic acid) [19]
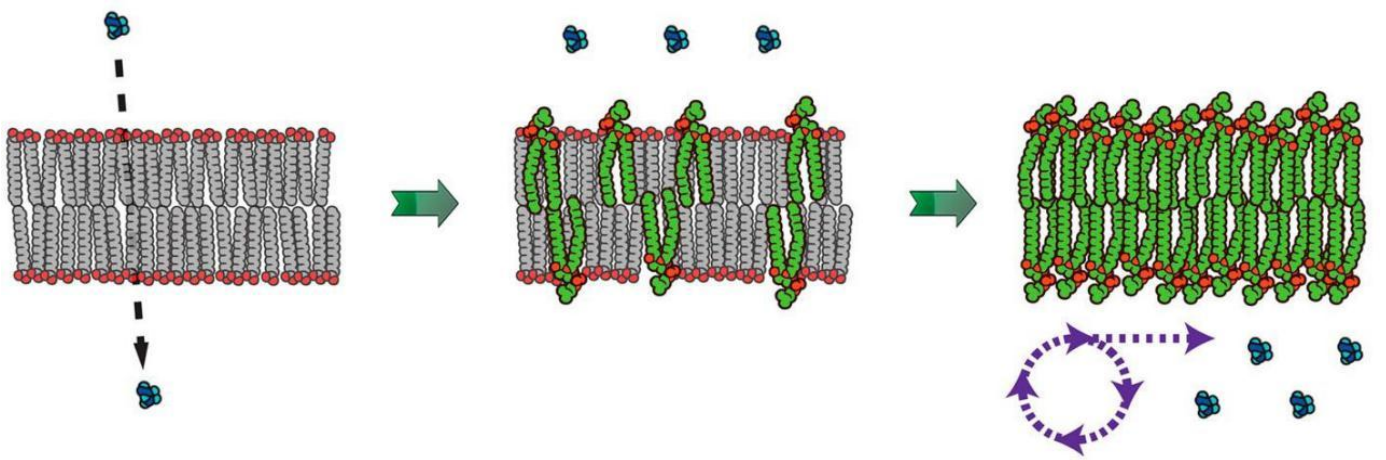

Figure 11. Schematic for membrane-driven cellular evolution; the gradual transition from highly permeable primitive membranes (left) to phosopholipid bilayer (right) which is

It is thought that the first ribosome every to have evolved is a simple Acyl transfer ribozyme capable of forming phospholipids which are then incorporated into the fatty acid membrane, increasing the overall polarity of the membrane and Donan effect that membrane has on neighboring ones, in other words increasing its competition and natural selection. Evolution would eventually cause strains on metabolic reactions to have developed inside the primitive cell now that its ions would not leak out and it could make use of the energy it has localized (Figure 11) [20].

Driven by the selective growth advantageous provided by increasing phospholipid content in the membrane. In turn, this transition in membrane composition imposes a selective pressure for the emergence of internalized metabolism to counter the reduced permeability of diacyl lipid membranes [19].

\section{The Challenges and Synthetic Solutions for Chemical Replication of RNA}

Countless challenges are faced by a RNA self-replicating cycle; for it to be a fully chemically and enzymatically free reaction, the cycle loses rate and fidelity, so much that it does not even reach critical threshold for the sustenance of life, meaning the RNA nucleotides break apart faster than the incorporation of nucleotides takes place, thus is the case when experimenting with modern substrates, that do not leak out of cells and are very polar with the regularly known triphosphate ester, this is advantageous to the modern cell where it uses enzymes to catalyze the release of di-phosphate, but not for the primitive cell as the substrates are found in the environment and require continuous dynamic exchange [19].

\section{Phosphoramidites}

Activated nucleotides are synthesized by the naturally degrading enzyme phosolyase, by breaking of already polymerized nucleotides. These nucleotides do not have the very polar triphosphate group, but rather a monophosphate group with a cyclic compound that reduces its polarity (Figure 8 ) and allows it to move across membranes more efficiently as would be the prebiotic case.

Monomer concentration, purity, their hydrolysis and self-cyclization cause the rate and fidelity to drastically drop below critical threshold, also when mismatched Watson-Crick base pairs occur a phenomenon known as 
the stalling effect slows down the rate and thus overall fidelity of the reaction. Other issues such as the region-specificity of the polymer linkage come to mind as well, Sulston in 1968 showed the abundance of the more natural 3' -5 ' phosphodiester linkage is lower than expected, the more abundant is the not so natural 2'- 5' [23].

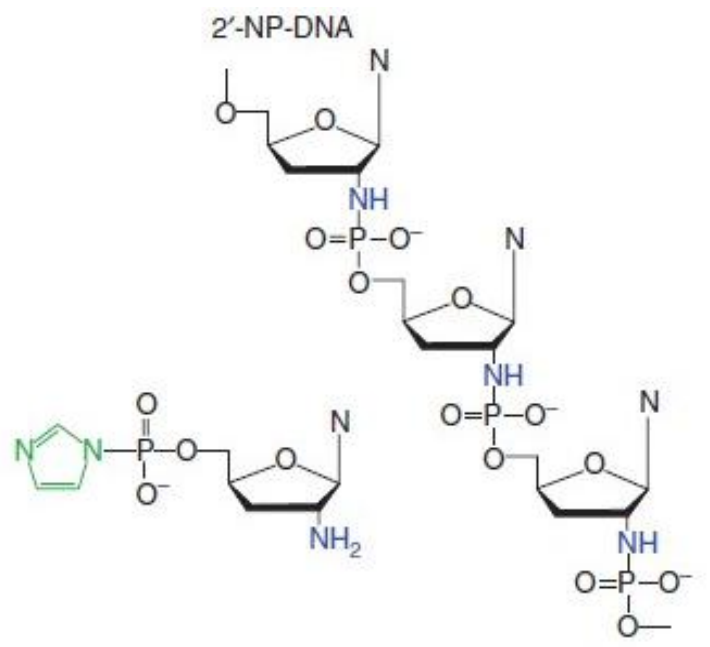

Figure 12. Structures of the 2'-5' phosphoramidate DNA; and it's corresponding activated monomer [9].

Reactivation chemistry; during the early stages of study on template replication and polymerization of nucleotide derivatives, a study by Orgel and Lohrmann in 1976, showed that by replacing the sugar hydroxyl in the monomer with a more nucleophilic amino group (Figure 8), a more rapid nucleophilic binding reaction occurs $\mathrm{N} 3$ ' $\triangle \mathrm{P} 5$ ', that overall increases monomer activity [24], these monomers participated in short oligonucleotide primer copying from 5-13 nucleotides in length, shown in the works of Tohidi in 1987 [25]. Phosophoramidites (nucleotide derivatives) (Figure 8) were thus taken into consideration for the study of primitive non enzymatic replication reactions, although they may have not been prebiotically available, they are still our best assumptions when trying to overcome the critical threshold of rate and fidelity, this higher reactivity rate comes at the price of faster intra-molecular monomer cyclization which would eventually deplete the template copying system of the activated nucleotides [26]. An example of the structures of the 2'-5' phosphoramidate DNA and its corresponding activated monomer can be seen in the works of Schrum (Figure 12) [9].

\section{Alternative Experiments}

Experiments carried out on alternatives to the ribose and deoxyribose sugar back bones show a particularly interesting candidate; TNA (Threose Nucleic Acid), a 5 atom back bone which forms standard Watson-Crick duplexes [27], the resulting decrease in flexibility of the duplex is now being studied to find a link to overall fidelity of template copying reactions.

Some metallic ions have also been used to increase region-specificity towards the more natural 3'- 5' linkage, Sleeper and Orgel in 1979 showed that $\mathrm{Pb}^{2+}$ or $\mathrm{UO}_{2}{ }^{2+}$ are capable of driving polymerization to form more 3'- 5' phosphoester linkage, but also showed that the 2'- 5' linkage occurs inevitably [28].

Ertem and Ferris in 1997 showed that polymerization of activated nucleotides from heterogenous templates constrained between the surfaces of a clay element known as montmorillonite was sufficient enough to form 3'- 5' linkage RNA molecules, these results pleased researchers as montmorillonite is an abundant element on earth and is believed to have been available on primitive earth [29].

More interestingly, Kanavarioti, Monnard and Deamer in 2001 showed the same linkage can be achieved if these activated nucleotides were placed in water and allowed to freeze; when the aqueous solution freezes the solutes become concentrated in between the ice crystals, and is sufficient enough to polymerize the activated nucleotides into RNA chains with random base pairing, they went on to show that the presence of metal ions in dilute solutions frozen at -18 degrees $\mathrm{C}$ to form oligouridylates 11 bases long with a product yield that typically exceeded $90 \%$, this information supports the probability of random RNA assembly in exo-planets, although the origins and the state at which these activated nucleotides or polymeric compound were/are in is still in debate [30].

Self-replicating RNA enzymes form a replication cycle that could involve two or more paired ribozymes (Figure 11), these systems can replicate and divide given that the coribozymes are both present in addition to their substrates (Figure 13) [4]. 

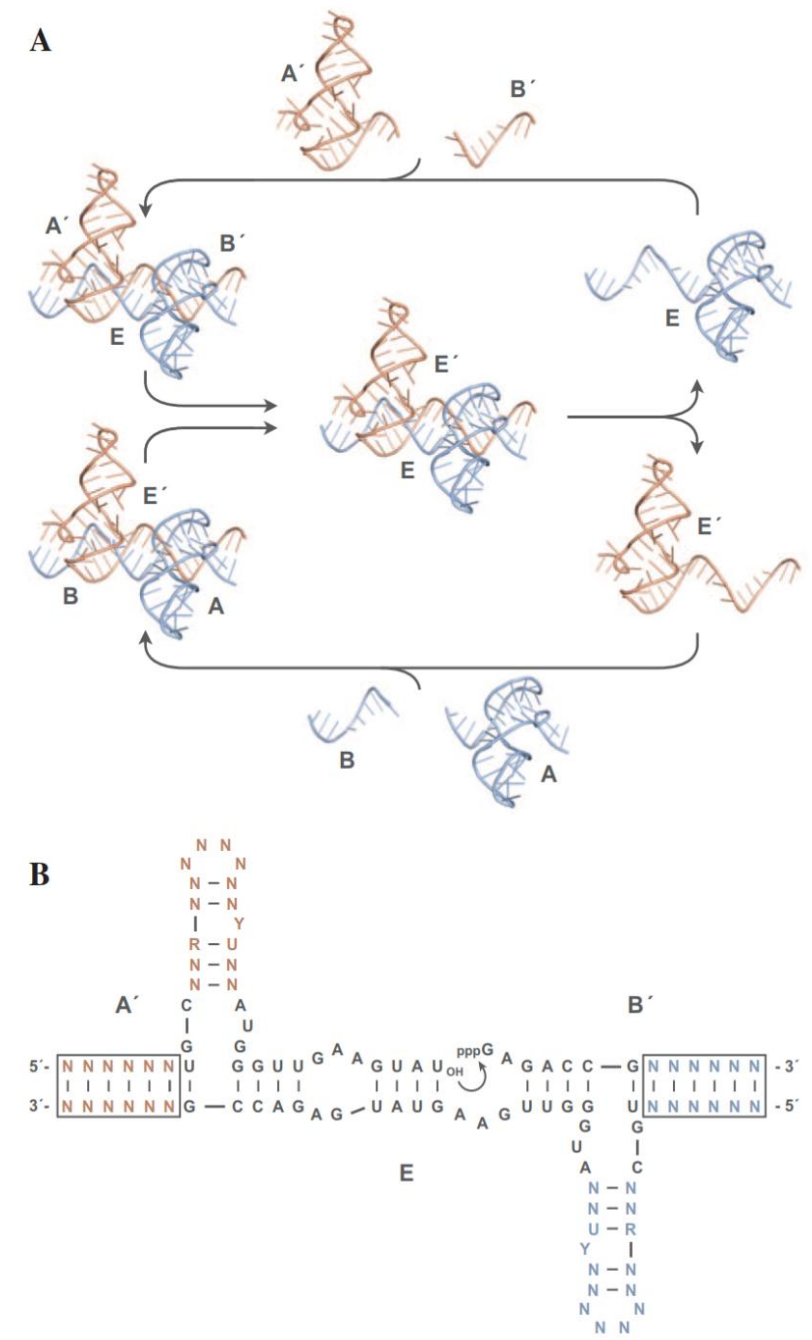

Figure 13. Self-replicating RNA enzymes; the figure depicts a schematic representation of a replication cycle involving a paired ribozyme, E ; E', with oligonucleotide substrates A'+B'; A+B respectively [4]

\section{Horizontal Gene Transfer and the Loss of Genealogical Records}

To understand the RNA world, a key concept known as Horizontal Gene Transfer (HGT) must be explained, this phenomenon has long been known, but the HGT we thought we knew is not the HGT that genomic revealed [2], [3].
HGT has the capacity to affect the entire genome and therefore over time erase an organism's genealogical trace; making HGT in evolutionary power and consequence comparable to the classical vertical evolutionary mechanisms [3].

Although organisms do have a genealogical defining core, which is traced back to the root of the universal phylogenetic tree (Figure 1), the core still remains very small due to our classical motivated thinking reflecting only the common history between the majority of genes (which are historical common). What would be the case of an organism that does not share a single common historical gene? This question again goes beyond the classical Darwinian concepts and tries to address the RNA world appropriately [3].

The most notable characteristic of HGT is the extreme variation in its frequency and phylogenic range from one gene to another; in the late 1990's it became possible to produce universal phylogenetic trees that were based on molecules other than rRNA; these showed that the majority of other molecules examined differed significantly in topology from the rRNA tree. The many protein trees that differ in topology from the rRNA tree also differ from one another, which is the most notable characteristic of HGT [1], [2].

Koonin and colleagues compared the topologies of approximately 7000 trees of the most commonly occurring bacterial and archaeal genes, and found that despite the wide spread variation of tree topologies, the trees of approximately 100 genes are universal and almost all of them encoding translation system requirements [1]. This indicates that translation was one of the first systems to evolve and not be lost be HGT.

HGT is based on the cellular componentry of the cell (donor or recipient), which can be classified according to their degree of connectedness to the cell; large, self-defining structures and functions that are loosely connected to the cell with minimal interactions with other elements are good candidates of HGT as alien homologues may completely replace them. While on the other hand, tightly coupled elements which have an extensive and specific tie to other elements (both physically and chemically) are hard to be replaced by an alien homologue. HGT is also characterized by high genetic temperature, as this genetic temperature drops genetic complexity rises and could give birth to the vast diverse phylogenetic trees [1]. 


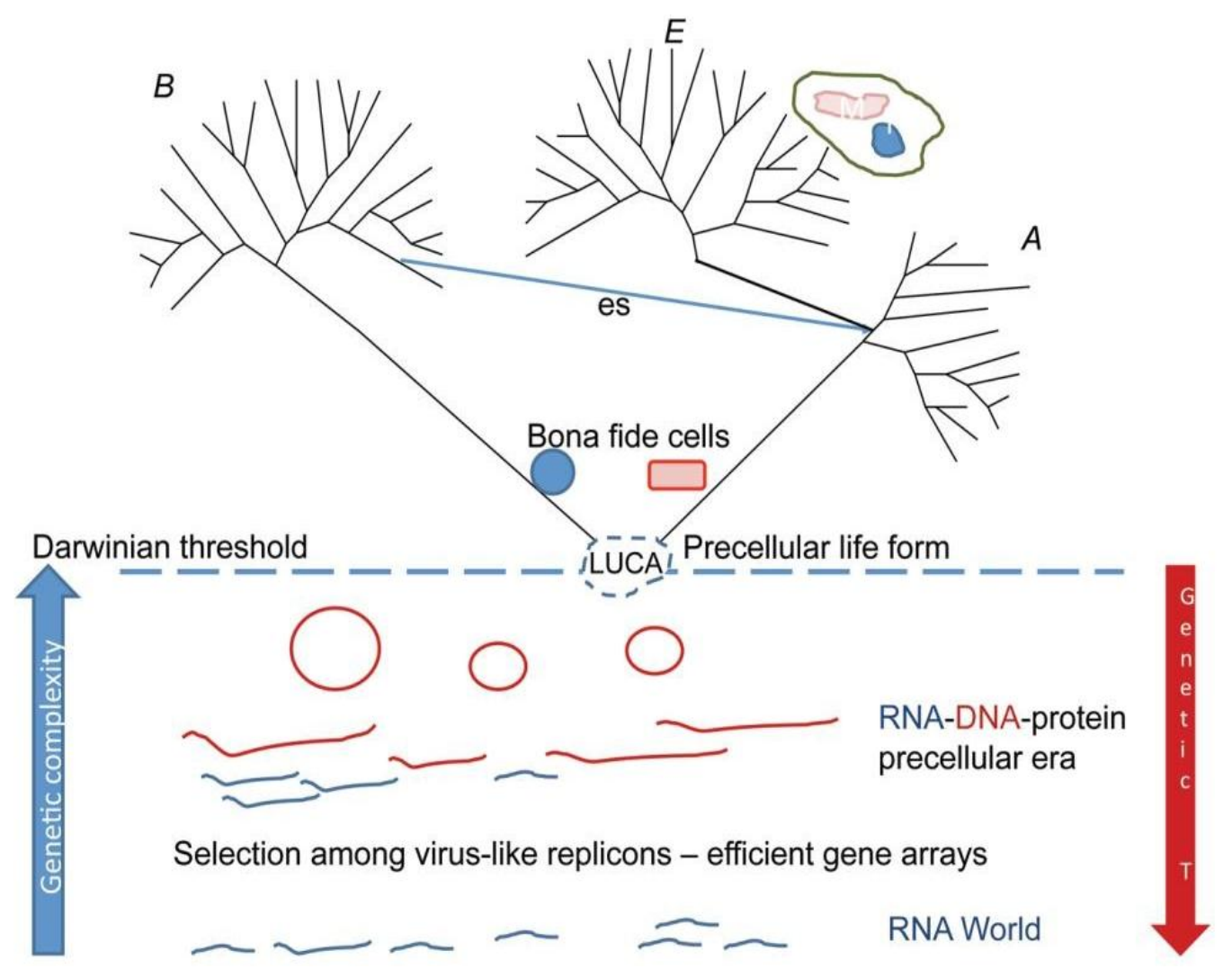

Figure 14. Scenario of Genetic complexity vs Genetic temperature; as Genetic temperature drops, genetic complexity rises. (a) archea, (b) bacteria, (e) eukaryotes, (es). Endosymbiosis which is considered to have occurred by engulfment of an a-proteobacterium by an archaeon of the TACK superphylum (filled blue line), Darwinian threshold which gives rise to Bona fide cells (dashed blue line), and the Last Universal Common (Cellular) Ancestor (LUCA) which is envisioned as a primitive life-form and possibly have a porous membrane [1] [31]

\section{Criticism}

Countless objections have been raised on the RNA world hypothesis, and in the end no true observations can be made, the fossils left behind by the RNA world weren't geologically significant like those left behind by the dinosaurs, rather traces of molecular existence of an RNA world which also still remain as assumptions. The RNA world hypothesis will forever be unproven, because direct observation of the issue is just impossible. Different annalistic views of the data have yielded alternative theories such as the 'Protein first' theory, which states that proteins developed prior to- or at least co-developed along with nucleic acids, the most obvious criticisms addressed; (i) RNA is too complex a molecule to have risen pre-biotically; (ii) RNA is inherently unstable; (iii) Catalytic functions are relatively rare properties of long RNA sequences only; and (iv) the catalytic range of RNA is too limited [32].

\section{RNA is Complex}

RNA is an extremely complex molecule, with four different nitrogen-containing heterocycles (AUCG) hanging of a backbone of alternating phosphate and D-ribose groups linked by 3'-5' linkages. Although a number of problems with its pre-biotic synthesis described in chapter III - have been addressed; it still remains that RNA is too complex a molecule to have risen pre-biotically, because as an issue (such as fidelity) is addressed, another issue (such as activation energy) arises [32]. It would be unmanageable to address all issues of RNA complexity and still retain its pre-biotic pathway to evolution.

\section{RNA is Inherently Unstable}

RNA is often considered too unstable to have accumulated in the prebiotic environment. RNA is particularly liable at moderate to high temperatures, and thus a number of researchers have suggested that the RNA world evolved in ice or in eutectic phases, but at such temperatures the RNA sequences have an increased tendency to base pair leading to the formation of unwanted intermolecular complexes that eventually reduce catalytic activity of the RNA sequence. A further problem of stability is caused by the susceptibility of RNA to be hydrolyzed by base-catalyzed hydrolysis at $\mathrm{pH}>6$, limiting RNA evolution to acidic environments of $\mathrm{pH}$ 4-5 [32].

While $\mathrm{Mg}^{2+}$ is important for stabilizing RNA secondary and tertiary structures (Hairpin loops or Hammer heads), 
high $\mathrm{Mg}^{2+}$ concentrations may also catalyze RNA degradation, here also the acidic environment that RNA is limited to plays a role, as the positive charge on protonated cytosine and adenosine residues decrease the need of divalent cation stabilizers such as $\mathrm{Mg}^{2+}$ [32].

\section{Catalysis is a Rare Function of RNA}

The RNA world hypothesis has been criticized mostly because of the belief that long RNA sequences are needed for the catalytic function of RNA. These long sequences are enormous and are needed to isolate the catalytic and biding functions of the overall ribozyme (for representative example see Figure 7) [32].

For example the best ribozyme replicase created so far, which is able to replicate an impressive 95-nucleotide stretch of RNA, is $~ 190$ nucleotides in length, which is by far too large a number to have risen in any random assembly, thus in vitro selection experiments had to be designed where $10,000,000,000,000$ $1,000,000,000,000,000$ of randomized RNA molecules are required as the starting point for the isolation of ribozymic and/or binding activity. This experiment clearly contradicts the probable prebiotic situation [32].

\section{The Catalytic Range of RNA is too Limited}

It has been proposed that the metabolic requirements needed for an RNA world to persist would have exceeded the catalytic capacity of RNA. The majorities of naturally occurring ribozymes catalyze phosphoryl transfer reactions, and at a comparable rate to protein enzymes of one million times weaker, presumably because proteins are composed of 22 chemically different amino acids as opposed to 4 very similar nucleotides [32].

\section{Conclusions}

The RNA world hypothesis has been stated; the start and birth of the RNA world ideology has been explained, including the time line of assumed events and the assumptions of cellular and molecular structure and function, in addition to chemical challenges faced by self-replicating systems and the struggle of loss of genealogical records caused by HGT.

From our knowledge of present-day organisms and the molecules they contain, it seems likely that the development of the directly autocatalytic mechanisms fundamental to living systems began with the evolution of families of molecules that could catalyze their own replication. With time, a family of cooperating RNA catalysts probably developed the ability to direct synthesis of polypeptides.

The hypothesis of an early RNA World is firmly rooted in empirical data and is part of a long and storied scientific perspective that goes back more than fifty years when the discovery of the centrality of RNA and ribonucleotides in protein synthesis and biochemical reactions took place.

To conclude that too many criticisms and errors arise in the RNA world model, the studies on this model are completely biased towards it, and the articles criticizing the model are also biased against it. Such a model is not valid for the origin of cellular life.

\section{REFERENCES}

[1] E. V. Koonin, 'Carl Woese's vision of cellular evolution and the domains of life', RNA Biol., vol. 11, no. 3, pp. 197-204, 2014, doi: 10.4161/rna.27673.

[2] C. R. Woese, 'On the evolution of cells', Proc. Natl. Acad. Sci., vol. 99, no. 13, pp. 8742-8747, Jun. 2002, doi: 10.1073/pnas.132266999.

[3] C. R. Woese, 'Interpreting the universal phylogenetic tree', Proc. Natl. Acad. Sci. U. S. A., vol. 97, no. 15, pp. 8392 8396, Jul. 2000, doi: 10.1073/pnas.97.15.8392.

[4] G. F. Joyce, 'Bit by bit: the Darwinian basis of life', PLoS Biol., vol. 10, no. 5, p. e1001323, 2012, doi: 10.1371/journal.pbio.1001323.

[5] M. de la Peña and I. García-Robles, 'Ubiquitous presence of the hammerhead ribozyme motif along the tree of life', $R N A$ N. Y. N, vol. 16, no. 10, pp. 1943-1950, Oct. 2010, doi: 10.1261/rna.2130310.

[6] G. F. Joyce and J. W. Szostak, 'Protocells and RNA Self-Replication', Cold Spring Harb. Perspect. Biol., vol. 10, no. 9, p. a034801, Sep. 2018, doi: 10.1101/cshperspect.a03 4801 .

[7] K. E. Olson, G. F. Dolan, and U. F. Müller, 'In vivo evolution of a catalytic RNA couples trans-splicing to translation', PloS One, vol. 9, no. 1, p. e86473, 2014, doi: 10.1371/journal.pone.0086473.

[8] R. P. D. Bank, 'RCSB PDB - 4QVT: Crystal structure of predicted $\mathrm{N}$-acyltransferase (ypeA) in complex with acetyl-CoA from Escherichia coli'.https://www.rcsb.org/str ucture/4qvt (accessed Dec. 31, 2020).

[9] J. P. Schrum, T. F. Zhu, and J. W. Szostak, 'The Origins of Cellular Life', Cold Spring Harb. Perspect. Biol., vol. 2, no. 9, Sep. 2010, doi: 10.1101/cshperspect.a002212.

[10] W. F. Doolittle, 'The spliceosomal catalytic core arose in the RNA world... or did it?', Genome Biol., vol. 14, no. 12, p. 141, Dec. 2013, doi: 10.1186/gb4145.

[11] G. F. Joyce, 'The antiquity of RNA-based evolution', Nature, vol. 418, no. 6894, pp. 214-221, Jul. 2002, doi: $10.1038 / 418214 a$.

[12] 'The difficult case of an RNA-only origin of life'. https://www.ncbi.nlm.nih.gov/pmc/articles/PMC7289000/ (accessed Feb. 07, 2021).

[13] W. Ma, 'The scenario on the origin of translation in the RNA world: in principle of replication parsimony', Biol. Direct, 
vol. 5, p. 65, Nov. 2010, doi: 10.1186/1745-6150-5-65.

[14] A. Geiger, P. Burgstaller, H. von der Eltz, A. Roeder, and M. Famulok, 'RNA aptamers that bind L-arginine with sub-micromolar dissociation constants and high enantioselectivity', Nucleic Acids Res., vol. 24, no. 6, pp. 1029-1036, Mar. 1996, doi: 10.1093/nar/24.6.1029.

[15] M. P. Robertson and G. F. Joyce, 'The Origins of the RNA World', Cold Spring Harb. Perspect. Biol., vol. 4, no. 5, May 2012, doi: 10.1101/cshperspect.a003608.

[16] G. F. Joyce and L. E. Orgel, '1 Prospects for Understanding the Origin of the RNA World', Cold Spring Harb. Monogr. Arch., vol. 24, no. 0, Art. no. 0, Jan. 1993, doi: $10.1101 / 0.1-25$

[17] 'The Origin of Prebiotic Information System in the Peptide/RNA World: A Simulation Model of the Evolution of Translation and the Genetic Code'.https://www.ncbi.nlm .nih.gov/pmc/articles/PMC6463137/ (accessed Feb. 07, 2021).

[18] S. S. Mansy, J. P. Schrum, M. Krishnamurthy, S. Tobé, D. A. Treco, and J. W. Szostak, 'Template-directed synthesis of a genetic polymer in a model protocell', Nature, vol. 454, no. 7200, pp. 122-125, Jul. 2008, doi: 10.1038/nature07018.

[19] I. Budin and J. W. Szostak, 'Physical effects underlying the transition from primitive to modern cell membranes', Proc. Natl. Acad. Sci. U. S. A., vol. 108, no. 13, pp. 5249-5254, Mar. 2011, doi: 10.1073/pnas.1100498108.

[20] T. F. Zhu and J. W. Szostak, 'Coupled growth and division of model protocell membranes', J. Am. Chem. Soc., vol. 131, no. 15 , pp. 5705-5713, Apr. 2009, doi: 10.1021/ja900919c.

[21] I. Budin and N. K. Devaraj, 'Membrane assembly driven by a biomimetic coupling reaction', J. Am. Chem. Soc., vol. 134, no. 2, pp. 751-753, Jan. 2012, doi: 10.1021/ja2076873.

[22] Y. Lyu et al., 'Protocells programmed through artificial reaction networks', Chem. Sci., vol. 11, no. 3, pp. 631-642, Jan. 2020, doi: 10.1039/C9SC05043D.

[23] J. Sulston, R. Lohrmann, L. E. Orgel, and H. T. Miles, 'Nonenzymatic synthesis of oligoadenylates on a polyuridylic acid template', Proc. Natl. Acad. Sci. U. S. A., vol. 59, no. 3, pp. 726-733, Mar. 1968, doi: 10.1073/pnas.59.3.726.

[24] R. Lohrmann and L. E. Orgel, 'Template-directed synthesis of high molecular weight polynucleotide analogues', Nature, vol. 261, no. 5558, pp. 342-344, May 1976, doi: $10.1038 / 261342 \mathrm{a} 0$

[25] M. Tohidi, W. S. Zielinski, C. H. Chen, and L. E. Orgel, 'Oligomerization of 3'-amino-3'deoxyguanosine-5' phosph orimidazolidate on a $\mathrm{d}(\mathrm{CpCpCpCpC})$ template', J. Mol. Evol., vol. 25, pp. 97-99, 1987, doi: 10.1007/BF02101750.

[26] A. R. Hill, L. D. Nord, L. E. Orgel, and R. K. Robins, 'Cyclization of nucleotide analogues as an obstacle to polymerization', J. Mol. Evol., vol. 28, no. 1-2, pp. 170-171, Feb. 1988, doi: 10.1007/BF02143509.

[27] K. Schöning, P. Scholz, S. Guntha, X. Wu, R Krishnamurthy, and A. Eschenmoser, 'Chemical etiology of nucleic acid structure: the alpha-threofuranosyl-(3'-->2') oligonucleotide system', Science, vol. 290, no. 5495, pp. 1347-1351, Nov. 2000, doi: 10.1126/science.290.5495.134 7.

[28] S. Hl, L. R, and O. Le, 'Template-directed synthesis of oligoadenylates catalyzed by $\mathrm{Pb} 2+$ ions.', J. Mol. Evol., vol. 13, no. 3, pp. 203-214, Oct. 1979, doi: 10.1007/bf01739480.

[29] G. Ertem and J. P. Ferris, 'Template-directed synthesis using the heterogeneous templates produced by montmorillonite catalysis. A possible bridge between the prebiotic and RNA worlds', J. Am. Chem. Soc., vol. 119, no. 31, pp. 7197-7201, Aug. 1997, doi: 10.1021/ja970422h.

[30] A. Kanavarioti, P. A. Monnard, and D. W. Deamer, 'Eutectic phases in ice facilitate nonenzymatic nucleic acid synthesis', Astrobiology, vol. 1, no. 3, pp. 271-281, 2001, doi: $10.1089 / 15311070152757465$.

[31] E. V. Koonin, M. Krupovic, S. Ishino, and Y. Ishino, 'The replication machinery of LUCA: common origin of DNA replication and transcription', BMC Biol., vol. 18, no. 1, p. 61, Jun. 2020, doi: 10.1186/s12915-020-00800-9.

[32] H. S. Bernhardt, 'The RNA world hypothesis: the worst theory of the early evolution of life (except for all the others)(a)', Biol. Direct, vol. 7, p. 23, Jul. 2012, doi: 10.1186/1745-6150-7-23. 